CLINICAL STUDY

\title{
A new functional parameter measured at the time of ablation that can be used to predict differentiated thyroid cancer recurrence during follow-up
}

\author{
Robbert B T Verkooijen ${ }^{1}$, Daphne Rietbergen ${ }^{1}$, Jan W Smit ${ }^{2}$, Johannes A Romijn ${ }^{2}$ and Marcel P M Stokkel ${ }^{1}$ \\ Departments of ${ }^{1}$ Nuclear Medicine and ${ }^{2}$ Endocrinology, Leiden University Medical Center, PO Box 9600, 2300 RC Leiden, The Netherlands \\ (Correspondence should be addressed to M P M Stokkel; Email: m.p.m.stokkel@lumc.nl)
}

\begin{abstract}
Background: This study addresses the questions whether patients with a high risk for recurrent thyroid cancer can be identified at initial stage, i.e. at the time of ablation.

Methods: We evaluated tumor recurrence in consecutive patients treated for differentiated thyroid cancer (DTC). Prognostic factors were statistically analyzed. We defined prognostic parameters based on thyroglobulin (Tg) levels, 24-h I-131 uptake rates and TSH values: (a) Tg/TSH, (b) Tg/24-h I-131 uptake value, and (c) Tg/(TSH $\times 24-h$ I-131 uptake).

Results: We included 190 patients (50 male, 140 female; mean age 47 years) with DTC for analysis, 146 without distant metastases and 44 with M1 tumor stage at initial presentation. The mean period of follow-up was 10.4 years (s.D. \pm 3.7 years). In 18 out of the 146 DTC patients with MO disease $(12.4 \%)$, tumor recurrence was found during follow-up. Although tumor stage, age, and standard biochemical values significantly differ between patients with and without recurrent disease or between patients with M0 and M1 tumor stage, the newly defined parameter Tg/(TSH $\times 24-\mathrm{h}$ I-131 uptake) was the best independent significant prognostic parameter in the assessment whether patients will develop a tumor recurrence during follow-up or not.

Conclusion: High $\mathrm{Tg} /(\mathrm{TSH} \times 24-\mathrm{h}$ I-131 uptake) ratios justify an adjustment of the I-131 activity for ablation therapy. To assess the optimal cut-off value for a dose adjustment, however, further studies are required in more patients, but the initial results are encouraging with respect to improving outcome in DTC patients.
\end{abstract}

European Journal of Endocrinology 156 41-47

\section{Introduction}

Differentiated thyroid cancer (DTC) is a rather uncommon tumor with a high survival rate. The therapy of choice consists of (near-) total thyroidectomy followed by ablation with radioiodine-131 (I-131). This combined treatment schedule is a prerequisite for an optimal tumor destruction as well as for an optimal patient follow-up (1). The currently used follow-up strategy is based on regular thyroglobulin $(\mathrm{Tg})$ measurements under thyroid stimulating hormone (TSH) stimulation ( $\mathrm{Tg}$-off values) either using thyroid hormone withdrawal or rhTSH and I-131 whole body scintigraphy (WBS). Later on, $\mathrm{Tg}$ values without TSH stimulation may guide the clinician during the follow-up of DTC. The purpose of optimal follow-up protocols in thyroid carcinoma is the early detection of recurrent or metastatic thyroid cancer, as it has a great impact on morbidity and mortality. Indeed, patients with recurrent DTC have a less favorable prognosis than those with primary disease, as more than $50 \%$ of patients with a recurrence experience tumor-related mortality. One of the major issues in such cases is the fact that a tumor recurrence may loose the I-131 uptake capacity (2). In this respect, it has been stated that high ablation doses may be recommended to decrease the risk not only for persistent thyroid remnants but also for tumor recurrence. The question, however, could be raised whether this should be applied in all DTC patients or in subgroups only (3-6). Therefore, it would be helpful to have one single or a combination of prognostic parameters at initial stage that can be used to identify patients with a high risk for tumor recurrence during follow-up. Thyroglobulin measured at initial stage may be of prognostic value, but its value cannot be disconnected from the TSH stimulation and the amount of residual disease after surgery, expressed as the 24-h I-131 uptake value. In the present study, this value is a surrogate value for remnant size. Although ultrasonography or computed tomography (CT)-scanning of the head and neck region would give a better estimation of the residual thyroid volume after surgery than the uptake value, these imaging techniques are less reliable shortly after surgery due to edema. Furthermore, contrast administration is required for optimal CT-scanning of the neck which interferes with subsequent I-131 therapy. 
In the present study, data from 190 patients with newly diagnosed DTC were analyzed in order to assess the prognostic value of commonly determined initial parameters in the prediction of a tumor recurrence during follow-up. Furthermore, we studied the prognostic value of three newly defined parameters which are based on Tg under endogenous TSH stimulation, TSH values, and/or the 24-h I-131 uptake value, all measured at the time of ablation. With the results, we might be able to adjust the I-131 ablation activity to decrease the risk of a recurrence and to improve morbidity and mortality.

\section{Material and methods}

\section{Study population}

Data were collected from the records of consecutive patients with DTC who received ablation treatment with I-131 at the Leiden University Medical Center (LUMC) between January 1986 and December 1999. This included a total of 255 patients with pathologically verified DTC, i.e. either papillary, follicular, mixed papillary follicular, or follicular Hürthle carcinoma. For the current evaluation, a follow-up period of at least 1 year was required. Exclusion criteria were: missing biochemical parameters, the presence of Tg-antibodies (Tg-abs values $>50 \mu \mathrm{g} / \mathrm{l}$ ), or TSH levels $<20 \mathrm{U} / \mathrm{l}$ at the time of ablation $(n=49)$, unknown uptake $(n=17)$, and/or follow-up of $<1$ year $(n=10)$. As a result, 65 patients were excluded, resulting in a total study population of 190 patients. Out of these 190 patients, 146 had no distant metastases, whereas 44 had metastases at the time of initial diagnosis.

Hospital records were reviewed and the following (prognostic) data were recorded: age, gender, histopathological data, treatment characteristics, and laboratory values. Records of scintigrams were analyzed and coded. Tumor staging was scored according to the criteria of the fifth tumor node metastasis (TNM) Atlas. We defined three new parameters as follows: (i) $\mathrm{Tg} / 24-\mathrm{h}$ I-131 uptake, (ii) $\mathrm{Tg} / \mathrm{TSH}$, and (iii) $\mathrm{Tg} /(\mathrm{TSH} \times 24-\mathrm{h}$ I-131 uptake) (Tg is expressed in $\mu \mathrm{g} / \mathrm{l}$ and TSH in $\mathrm{U} / \mathrm{l}$ ).

\section{Radioiodine treatment}

Therapy for DTC consists of (near-) total thyroidectomy, followed 4-6 weeks later by radioiodine ablation therapy. During this interval, no treatment with L-thyroxin was initiated in order to increase TSH levels. The 24-h I-131 pre-treatment uptake value in the neck region was measured using standard techniques: $40 \mathrm{MBq}$ of I-131 was given orally, followed by planar scintigraphy of the neck region $24 \mathrm{~h}$ later. This uptake value is regarded as a surrogate value for remnant size in the present study. Although stunning may occur during diagnostic scanning with $\mathrm{I}-131$, a recent study by Dam et al. (7) demonstrated that treatment efficacy is not influenced by activities $<185 \mathrm{MBq}$ (7-9). In addition, follow-up studies and subsequent treatment are used to achieve a complete ablation. In case of a possible stunning effect, ablation failures will be depicted 6 months after initial treatment (see evaluation of treatment efficacy).

A standard I-131 activity of approximately $2800 \mathrm{MBq}$ was given orally $24 \mathrm{~h}$ after the uptake measurement and adjusted in case of large thyroid remnants. The rationale of this quantitative approach is to avoid unnecessary exposure and local radioiodine side effects. In this regimen, no adjustments were made in the case of cervical lymph node metastases. Treatment of patients with M1 tumor stage at initial presentation as well as subsequent treatment for either initial ablation failures or recurrent disease was done with $6100 \mathrm{MBq}$ of I-131. Seven days after each treatment, whole-body scans were made according to the protocol described below to assess loco-regional uptake and the presence of metastases.

\section{Evaluation of treatment efficacy}

Six months after the ablation therapy, L-thyroxin was withdrawn for at least 4 weeks. Subsequently, radioiodine diagnostic whole-body scintigrams were obtained 3 days after the administration of $185 \mathrm{MBq}$ of I-131 or $24 \mathrm{~h}$ after the administration of $370 \mathrm{MBq}$ I-123. For all scintigrams, a Toshiba gamma camera (Tokyo, Japan) was used. A high-energy collimator (matrix sizes of $256 \times 1024$ and $256 \times 256$, window of $20 \%$ centered at $360 \mathrm{keV}$ ) for the I-131 WBS, a low-energy collimator (matrix sizes of $256 \times 1024$ and $256 \times 256$, window of $20 \%$ centered at $159 \mathrm{keV}$ ) for the I-123 WBS was used.

Anterior and posterior whole-body and planar views of the neck region were routinely obtained. For the whole-body scintigrams, scanning rates of 15 (I-131) and $10 \mathrm{~cm} / \mathrm{min}$ (I-123) were used. In addition, Tg values were determined at the time of the diagnostic whole-body scan to document the ablation efficacy. For successful ablation, a cut-off level of $\mathrm{Tg}$ of $\leq 1 \mu \mathrm{g} / \mathrm{l}$ was applied. Patients with an unsuccessful ablation, documented by scintigraphy and/or a $\mathrm{Tg}$ value $>1 \mu \mathrm{g} / \mathrm{l}$ during TSH stimulation after 4 weeks of L-thyroxin withdrawal, received a second treatment with I-131.

Recurrent disease was defined as increased $\mathrm{Tg}$ levels, abnormal WBS, or both but not within 2 years after ablation and following at least one diagnostic session with normal test results. Persistent disease or ablation failures were defined if one or both tests remained abnormal after ablation, irrespective of the time interval.

\section{Analytical methods}

Until 1997, serum Tg was measured using an IRMA, the Dynotest TG (Brahms Diagnostica GmBH, Germany), with a detection limit of $1 \mu \mathrm{g} / \mathrm{l}$. From 1997 onwards the Dynotest TG-s (Brahms Diagnostica 
$\mathrm{GmBH})$ was used, with a detection limit of $0.5 \mu \mathrm{g} / \mathrm{l}$. Recurrent disease, however, was defined as Tg levels $>$ $1 \mu \mathrm{g} / \mathrm{l}$. TSH levels were measured by means of an immunofluorometric assay (IFMA) with the Delfia (Wallac, Turku, Finland) until 1997. Thereafter, an immunoluminometric assay (ILMA) was used with the Elecsys (Boehringer Mannheim, Germany). Serum Tg-abs were determined by the Ab-HTGK-3 IRMA test (DiaSorin Biomedics, Italy).

\section{Data collection and statistical analysis}

All collected data were put in a database using MS-Access 2000. Statistical analysis was performed with SPSS 11.5 for Windows (SPSS Corporation, Chicago, IL, USA) and MS-Excel 2000. The quantitative data were analyzed using Cross-tabs with $\chi^{2}$, Student's $t$-test, Cox regression, Cox regression forward stepwise, and by calculating curve coordinates. The prognostic value of the patient characteristics was quantified with the hazard ratio and its 95\% confidence level. Throughout, a $P$ value of 0.05 or less was considered statistically significant. Finally, three subgroups were identified for statistical analysis: patients with initially MO stage DTC, irrespective of recurrent disease during follow-up; patients with initially M1 stage DTC; and finally, patients with initially MO stage disease, but with recurrent DTC during follow-up. For the generation of the probability plot and prognostic stratification, only patients with initially MO disease were used, whereas their results were compared with patients with M1 disease to assess the overall value of the study results.

\section{Results}

In the present study, 190 patients (50 male, 140 female; mean age 47.1 years) with DTC were included for further analysis, 146 without distant metastases and 44 with M1 tumor stage at initial presentation. Papillary thyroid carcinoma, consisting of papillary and mixed papillary follicular, was diagnosed in 102 patients. Follicular thyroid carcinoma was diagnosed in 44 patients. In the present study, 49 patients had lymph node involvement at the time of thyroidectomy. The mean period of follow-up was $10.4 \pm 3.7$ years. In 18 out of the 146 DTC patients with M0 disease (12.4\%), tumor recurrence was found during follow-up. A total of 14 patients died in the group without metastases, 6 from the differentiated thyroid carcinoma, whereas 8 died from other causes, such as second primary tumors.

Table 1 shows subgroup characteristics of patients with initially M0 stage $(n=146)$ and patients with M1 stage $(n=44)$ DTC as well as for patients with initially M0 stage without $(n=128)$ and with $(n=18)$ recurrent disease respectively. Significant differences were found between almost all parameters tested between patients with M0 and M1 initial tumor stage. For age, we found a cut-off level of 59 years between those with a high and low risk for recurrent disease. In patients with MO disease, age at diagnosis ( $>59$ years), T stage, $\mathrm{N}$ stage, and all newly defined parameters were significantly different between those with and without tumor recurrence during follow-up. For the risk of recurrent disease, Tg levels $(P=0.027)$, N stage $(P=0.038)$, and $\mathrm{Tg} / \mathrm{TSH}(P=0.021)$ were significant correlates for

Table 1 Differences between differentiated thyroid cancer patients with and without metastases and between patients with and without recurrent disease during follow-up.

\begin{tabular}{|c|c|c|c|c|c|c|}
\hline & $\begin{array}{l}\text { Patients with } \\
\text { initially M0 } \\
\text { (s.D.) }(n=146)\end{array}$ & $\begin{array}{l}\text { Patients with } \\
\text { initially M1 } \\
\text { (s.D.) }(n=44)\end{array}$ & $P$ value & $\begin{array}{l}\text { M0 without RD } \\
\text { (s.D.) }(n=128)\end{array}$ & $\begin{array}{l}\text { M0 with RD } \\
\text { (s.D.) }(n=18)\end{array}$ & $P$ value \\
\hline Gender: male/female & $32 / 114$ & $13 / 31$ & 0.400 & $27 / 101$ & $5 / 13$ & 0.521 \\
\hline Age at diagnosis (years) & $42.6( \pm 14.87)$ & $61.9( \pm 16.46)$ & 0.001 & $41.5( \pm 14.02)$ & $50.7( \pm 18.3)$ & 0.013 \\
\hline Histology & & & 0.007 & & & 0.700 \\
\hline Papillary & 120 & 27 & & 104 & 16 & \\
\hline Follicular & 26 & 17 & & 24 & 2 & \\
\hline$T$ stage & & & 0.001 & & & 0.003 \\
\hline $\mathrm{T} 1$ & 7 & 1 & & 5 & 2 & \\
\hline T2 & 89 & 16 & & 85 & 4 & \\
\hline T3 & 25 & 5 & & 20 & 5 & \\
\hline T4 & 25 & 22 & & 18 & 7 & \\
\hline$N$ stage & & & 0.001 & & & 0.001 \\
\hline No & 110 & 31 & & 101 & 9 & \\
\hline N1 & 36 & 13 & & 27 & 9 & \\
\hline TSH (U/l) & $77 \pm 36$ & $62 \pm 17$ & 0.023 & $77 \pm 37$ & $78 \pm 34$ & 0.949 \\
\hline $\operatorname{Tg}(\mu \mathrm{g} / \mathrm{l})$ & $21 \pm 110$ & $1246 \pm 4066$ & 0.000 & $8 \pm 14$ & $120 \pm 321$ & 0.001 \\
\hline Uptake (\%) & $6.1( \pm 7.3)$ & $6.7( \pm 7.8)$ & 0.621 & $6.3( \pm 7.6)$ & $4.7( \pm 5.3)$ & 0.125 \\
\hline Tg/Uptake & $3.9( \pm 9.2)$ & $827.1(3312.3)$ & 0.003 & $2.5( \pm 5.6)$ & $15.3( \pm 19.9)$ & 0.001 \\
\hline $\mathrm{Tg} / \mathrm{TSH}$ & $0.4( \pm 2.3)$ & $51.2( \pm 207.9)$ & 0.003 & $0.1( \pm 0.2)$ & $2.7( \pm 6.6)$ & 0.001 \\
\hline $\mathrm{Tg} /(\mathrm{TSH} \times 24-\mathrm{h} \mathrm{I}-131$ uptake $)$ & $0.07( \pm 0.19)$ & $38.00( \pm 170.55)$ & 0.007 & $0.04( \pm 0.07)$ & $0.31( \pm 0.49)$ & 0.001 \\
\hline Initial ablation failure ${ }^{\mathrm{a}}$ & & & & $43(34 \%)$ & $8(44 \%)$ & 0.522 \\
\hline
\end{tabular}

$\mathrm{RD}$, recurrent disease; T stage, tumor stage; $\mathrm{N}$ stage, node stage; TSH, thyroid stimulating hormone; Tg, thyroglobulin.

${ }^{\mathrm{a}}$ Evaluation 6-12 months after the I-131 ablation therapy. 
tumor recurrence. In stepwise analysis, an increased ratio of $\mathrm{Tg} /(\mathrm{TSH} \times 24$-h I-131 uptake $)$ was selected as the most reliable variable $(P=0.001)$ for tumor recurrence (see Fig. 1), with a Hazard rate of 12.1 (95\% CI: 6.61-22.13). Initial ablation failure was not found to be a significant indicator for tumor recurrence. The number of patients with initial ablation failure was not significantly different between the groups with and without recurrent disease $(P=0.522)$.

To assess whether the parameters studied may be indicative for bulky disease, we compared the parameters of patients having initially M1 disease with those having initially MO disease and tumor recurrence during followup. In this respect, age at diagnosis was significantly different between these subgroups $(P=0.021$; see Table 2). Finally, we combined patients with M1 with patients with recurrent disease into one group to determine the accuracy of $\mathrm{Tg} /(\mathrm{TSH} \times 24-\mathrm{h} \mathrm{I-131}$ uptake) in the assessment of bulky disease at the time of ablation. The area under the receiver operating characteristic (ROC) curve was 0.800 (95\% CI: 0.714-0.866).

\section{Discussion}

In the present study, we have assessed the value of clinical and biochemical parameters in prognostic stratification of patients with DTC at the time of ablation with radioiodine. Although $\mathrm{T}$ stage, $\mathrm{N}$ stage, age, and standard biochemical values at the time of diagnosis significantly differ between patients with and without recurrent disease or between patients with M0 and M1 tumor stage, we found a considerable overlap between these parameters to be of clinical value. Nevertheless, current study revealed the newly defined parameter $\mathrm{Tg} /(\mathrm{TSH} \times 24-\mathrm{h}$ I-131 uptake) determined at initial stage to be the strongest independent significant prognostic factor for tumor recurrence during follow-up.

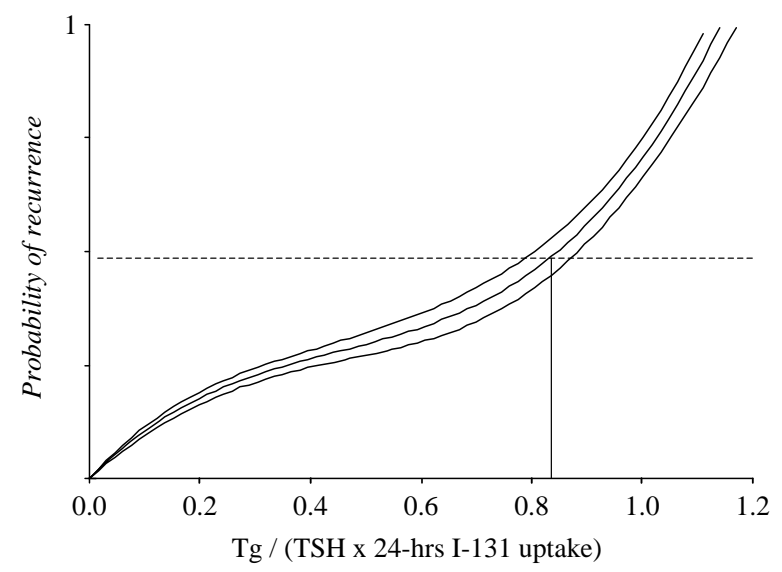

Figure 1 Probability plot of tumor recurrence with its $95 \%$ confidence interval in patients with initially M0 tumor stage. Tg, thyroglobulin; $\mathrm{TSH}$, thyroid-stimulating hormone.
The Leiden University Medical Center is a reference center for patients with relatively complicated DTC, which is reflected in the high number of patients $(n=$ 44) with M1 disease at initial stage. In $12 \%$ of the patients with initially MO disease tumor recurrence was seen during follow-up, which is in agreement with previous publications by others $(10-12)$. All patients had been treated by near-total thyroidectomy and subsequent ablation with radioiodine approximately 6 weeks after surgery. In a recent report (3), we have described that the use of rather low ablation doses results in failure rates up to $60 \%$ and, consequently, in a high number of additional treatments up to more than a year after initial operation. Although a relation between the initial treatment failure and recurrent disease was suggested, this could not be established in the present study, as differences in failure rates between patients with and without recurrent DTC were not statistically significant. This finding is in agreement with the data published by Falvo et al. (13).

In a recent report by Haigh et al. it was shown that survival of patients with DTC was not significantly influenced by the extent of initial thyroidectomy (14). The authors found 10-year survival rates of 72 and $78 \%$ respectively for total and partial thyroidectomy. In contrast to these results, however, Cushing et al. (15) reported on the prognostic value of the extension of initial surgery. They studied 333 patients with a mean age of 39.7 years and found that a total thyroidectomy revealed better results than a near-total or partial thyroidectomy. Regarding the number of patients with advanced disease at initial presentation in their study, it is not surprising that non-total thyroidectomy did not only result in a high frequency of large thyroid remnants, but also in a high number of patients with tumor remnants.

The fact that the presence of lymph node metastases is a predictive factor for recurrence, irrespective of initial surgery (partial or total thyroidectomy) (16), again underlines the issue that an optimal assessment and subsequent resection of the total tumor mass at initial stage is highly important for prognosis and survival. In addition, subsequent treatment with radioiodine, therefore, is almost a prerequisite for an optimal starting point, especially in extensive disease, as it destroys normal thyroid tissue as well as small tumor remnants. Indeed, Haq et al. (17) clearly showed the superior value of a combined therapeutic approach over surgery alone. Despite the low number of patients with recurrent disease in the present study, we found a significant difference in $\mathrm{N}$ stage between patients with and without recurrent disease $(P=0.001)$.

Different staging classifications have been proposed over the past decades in hopes of a better identification of high risk patients, i.e. patients with bulky disease. In this respect, the TNM tumor staging system is still the most commonly used system. Although it can be used to differentiate low-risk from high-risk patients, it was found to be less valuable in identifying intermediate-risk groups 
Table 2 Differences between differentiated thyroid cancer patients with initially distant metastases (M1) and with locoregional disease (M0) but a recurrence during follow-up.

\begin{tabular}{|c|c|c|c|}
\hline & $\begin{array}{l}\text { Patients with initially } \\
\text { M1 (s.D.) }(n=44)\end{array}$ & $\begin{array}{l}\text { Patients with initially } \\
\text { M0 and RD (S.D.) }(n=18)\end{array}$ & $P$ value \\
\hline Gender: male/female & $13 / 31$ & $5 / 13$ & 0.889 \\
\hline Age at diagnosis & $62 \pm 16$ & $51 \pm 18$ & 0.021 \\
\hline Histology & & & 0.061 \\
\hline Papillary & 27 & 16 & \\
\hline Follicular & 17 & 2 & \\
\hline$T$ stage & & & 0.149 \\
\hline $\mathrm{T} 1$ & 1 & 2 & \\
\hline $\mathrm{T} 2$ & 16 & 4 & \\
\hline T3 & 5 & 5 & \\
\hline $\mathrm{T} 4$ & 22 & 7 & \\
\hline$N$ stage & & & 0.217 \\
\hline No & 31 & 9 & \\
\hline N1 & 13 & 9 & \\
\hline TSH (U/I) & $62( \pm 17)$ & $78( \pm 34)$ & 0.225 \\
\hline $\operatorname{Tg}(\mu \mathrm{g} / \mathrm{l})$ & $1246( \pm 4066)$ & $120( \pm 321)$ & 0.276 \\
\hline Uptake (\%) & $6.7( \pm 7.8)$ & $4.7( \pm 5.3)$ & 0.311 \\
\hline Tg/Uptake & $827.1( \pm 3312.3)$ & $15.3( \pm 20.0)$ & 0.334 \\
\hline $\mathrm{Tg} / \mathrm{TSH}$ & $51.2( \pm 207.9)$ & $2.7( \pm 6.6)$ & 0.357 \\
\hline $\mathrm{Tg} /(\mathrm{TSH} \times 24-\mathrm{h} \mathrm{I}-131$ uptake $)$ & $38.00( \pm 170.55)$ & $0.31( \pm 0.49)$ & 0.383 \\
\hline
\end{tabular}

$\mathrm{RD}$, recurrent disease; T stage, tumor stage; $\mathrm{N}$ stage, node stage; TSH, thyroid stimulating hormone; Tg, thyroglobulin.

$(10,18)$. The age for men, gender and histological subtype (AMES) classification, a system based on age, gender, and histological parameters, as well as the European Organization for Research and Treatment of Cancer (EORTC) classification were both tested in a prognostic study on 499 DTC patients by Jukkola et al. (10), revealing that these methods were not reproducible. Finally, the metastasis, age, completeness of resection, local invasion, tumor size (MACIS) scoring classification, which is based on age, tumor size, incomplete surgery, extra-thyroidal invasion, and distant metastases, was found to leave the definition of the intermediate and high risk groups too wide. Consequently, most of the staging classifications are found to have more or less practical limitations.

One of the most well-known prognostic parameters is age at the time of diagnosis. In this respect, we observed not only a significant difference between patients with M0 disease (42.6 years) and those with M1 disease (61.9 years; $P=0.001$ ), but also between M0 tumor stage patients with (50.7 years) and without (41.5 years; $P=0.013$ ) recurrent disease. Falvo et al. (13) performed a study on biologically aggressiveness of DTC in elderly patients, in which an age exceeding 70 years represented the most unfavorable prognosis. Haq et al. (17) reported a comparable age of 70 years and older in relation to a poor outcome. Both Volante et al. (19) and Siironen et al. (20) described an age of $>45$ years in close relation to an aggressive tumor behavior. Based on the present data, however, it can be concluded that age at initial diagnosis is indicative for tumor extension, but it is not an independent prognostic parameter that can be used to identify patients with a high risk for tumor recurrence. Indeed, regarding age, the overlap between the subgroups defined is too much to be useful in clinical practice.
As tumor extension at initial presentation is highly important for the assessment of prognosis and clinical outcome, it would be helpful to have a test that better reflects tumor burden than current staging and imaging techniques. In one of the most interesting publications on this subject, a clear relation between tumor burden and thyroglobulin levels was described (21). The authors reported not only that the number of metastatic lesions was linked with serum Tg/TSH levels, but also their total volume. The diagnostic value of $\mathrm{Tg}$ was confirmed and established in subsequent reports and it was demonstrated that the value of stimulated Tg levels, i.e. values measured after hormonal withdrawal, clearly identifies persistent or recurrent disease. In a study by Giovanella et al. (11), post-surgery $\mathrm{Tg}$ levels above $4.5 \mu \mathrm{g} / \mathrm{l}$ identified $94 \%$ of patients with metastasis, which, according to the authors, could be taken into account in treatment planning. Bernier et al. showed that $\mathrm{Tg}$ values measured at the time of ablation and 5 days later expressed as TgD5/TgD0 ratio were highly suggestive for treatment failure (22). The larger the ratio, the better the final outcome. In other studies, Tg levels measured at 3 or 6 months after ablation were found to be strongly correlated with metastatic disease and the authors concluded that values measured at these time intervals are indicative for an intensive follow-up scheme or additional treatment $(23,24)$. Others have shown that the best positive predictive value for the detection of a local recurrence is brought by the slope of the Tg levels $(25,26)$. Finally, Kim et al. (27) showed that $\mathrm{Tg}$ levels measured at the time of immediate postoperative I-131 remnant ablation correlated well with serum $\mathrm{Tg}$ levels at the time of diagnostic whole-body scanning during follow-up. High Tg values 
were indicative for tumor persistence or recurrence of disease in the earliest postoperative period.

Under normal circumstances, i.e. in normal thyroid tissue, an optimal re-uptake and storage mechanism should prevent leakage of $\mathrm{Tg}$ into the bloodstream. In clinical practice, however, it has been found that this physiologic mechanism is not fulfilling this task completely with measurable $\mathrm{Tg}$ levels as a consequence in patients with normal function of the thyroid gland. Increased $\mathrm{Tg}$ production and a distortion of the physiological process, as seen in infection and malignancy, results in a misbalance and in increased serum $\mathrm{Tg}$ levels compared with normal circumstances, a phenomenon that is enhanced by TSH stimulation. Indeed, from data in literature, it is known that $\mathrm{Tg}$ measurements after hormonal withdrawal have a higher accuracy than Tg-on levels in the detection of malignancy. In this respect, a TSH stimulation $>20 \mathrm{U} / \mathrm{l}$ is regarded as prerequisite for an optimal $\mathrm{Tg}$ measurement. In general, patients who are admitted for ablation with radioiodine have normal thyroid tissue remnants in the neck region. Despite the optimal TSH stimulation at that time to facilitate an optimal I-131 uptake, $\mathrm{Tg}$ levels should be relatively low in the case of a normal function of the remnant, as the re-uptake and storage mechanisms are intact. On the other hand, the values may be significantly increased in remnants harboring malignant cells. In the present study, we have evaluated whether an increased Tg level is caused by a large nearly stimulated thyroid remnant or a small remnant with stimulated thyroid cancer cells. For this purpose, we have normalized the $\mathrm{Tg}$ levels for both the amount of functional thyroid tissue, expressed in a 24-h uptake value, and the amount of TSH stimulation. Although we used a low dose I-131 24-h uptake scan before therapy, it has become more and more common practice to perform a post-therapy scan with uptake measurements over the neck region. In this respect, one of the major issues for discussion is the possible stunning effect that may occur even when using activities of I-131 in the range of $40 \mathrm{MBq}(7-9)$. Since therapeutic activities of I-131 are nowadays more based on tumor stage instead of uptake values, post-treatment uptake values may be advised for prognostic stratification to avoid stunning effects by pre-treatment scans.

We found significant differences in $\mathrm{Tg}$ values at initial presentation between patients with (mean value, $8 \mu \mathrm{g} / \mathrm{l}$ ) and without (mean value, $120 \mu \mathrm{g} / \mathrm{l}$ ) recurrent disease during follow-up, whereas TSH stimulation and 24-h uptake values were not different. In the univariate analysis, $\mathrm{T}$ and $\mathrm{N}$ stage were significantly different between the two groups, but, using the stepwise forward multivariate analysis, $\mathrm{Tg} /(\mathrm{TSH} \times 24-\mathrm{h}$ uptake value $)$ remained the most significant independent parameter, irrespective of $\mathrm{N}$ and $\mathrm{T}$ stage. Moreover, except from age, we did not find significant differences in all parameters tested between patients with initially M1 disease and patients with MO stage demonstrating recurrent disease during follow-up. Even the newly defined parameters that were based on $\mathrm{Tg}$ values did not show any significance anymore, suggesting that high values indicate bulky DTC. Consequently, patients with MO stage and high $\mathrm{Tg} /(\mathrm{TSH} \times 24$-h uptake value) ratios at initial presentation should be regarded as patients with initially M1 disease and therefore be treated with higher I-131 doses. In addition, a close clinical follow-up scheme is recommended for these patients. Finally, it has to be realized that at severe malignancy the $\mathrm{Tg}$ production may be low and not high as found in the present study, which may cause a false negative effect on this new parameter. In such cases, radioiodine uptake is often also severely diminished, as the expression of the sodium-iodine symporter is commonly the first factor being affected in a dedifferentiation process of thyroid cancer. This condition, however, is very rare at initial presentation, whereas such features are more common in anaplastic tumors, which were excluded in the present study.

\section{Conclusion}

In the present study, we have evaluated the prognostic value of clinical and biochemical parameters measured at the time of ablation for DTC. Based on these values, we have defined a new parameter, $\mathrm{Tg} /(\mathrm{TSH} \times 24-\mathrm{h}$ uptake value), which was hypothesized to be a better prognostic indicator for the presence of malignant cells after total or near-total thyroidectomy for DTC. Although T stage, N stage, age at the time of diagnosis, and standard biochemical values significantly differ between patients with and without recurrent disease or between patients with M0 and M1 tumor stage, the newly defined parameter was the best independent significant prognostic parameter in the assessment whether patients will develop a tumor recurrence during follow-up or not. From the present data, it can be concluded that high ratios justify an adjustment of the I-131 doses as ablation dose and a close clinical follow-up.

\section{References}

1 Utiger RD. Follow-up of patients with thyroid carinoma. New England Journal of Medicine $1997 \mathbf{4 4} 493-495$.

2 Stojadinovic A, Shoup M, Nissan A, Ghossein RA, Shah JP, Brennan MF \& Shaha AR. Recurrent differentiated thyroid carcinoma: biological implications of age, method of detection, and site and extent of recurrence. Annals of Surgical Oncology 2002 9 789-798.

3 Verkooijen RBT, Stokkel MPM, Smit JWA \& Pauwels EKJ. Radioiodine-131 in differentiated thyroid cancer: a retrospective analysis of an uptake-related ablation strategy. European Journal of Nuclear Medicine and Molecular Imaging 200431 499-506.

4 Maxon HR III, Englaro EE, Thomas SR, Hertzberg VS, Hinnefeld JD, Chen LS, Smith H, Cummings D \& Aden MD. Radioiodine-131 
therapy for well-differentiated thyroid cancer - a quantitive radiation dosimetric approach: outcome and validation in 85 patients. Journal of Nuclear Medicine 199233 1132-1136.

5 Pacini F, Schlumberger M, Harmer C, Berg GG, Cohen O, Duntas J, Jamar F, Jarzab B, Limbert E, Lind P, Reiners C, Sanchez Franco F, Smit J \& Wiersinga W. Post-surgical use of radioiodine $\left({ }^{131} \mathrm{I}\right)$ in patients with papillary and follicular thyroid cancer and the issue of remnant ablation: a consensus report. European Journal of Endocrinology 2005153 651-659.

6 Pacini F, Schlumberger M, Dralle H, Elisei R, Smit JW \& Wiersinga W. European consensus for the management of patients with differentiated thyroid carcinoma of the follicular epithelium. European Journal of Endocrinology 2006154 787-803.

7 Dam HQ, Kim SM, Lin HC \& Intenzo CM. I-131 therapeutic efficacy is not influenced by stunning after diagnostic whole-body sanning. Radiology 2004232 527-533.

8 Lassmann M, Luster M, Hanscheid H \& Reiners C. Impact of I-131 diagnostic activities on the biokinetics of thyroid remnants. Journal of Nuclear Medicine 200445 619-625.

9 Kalinyak JE \& McDougall IR. Whole-body scanning with radionuclides of iodine, and the controversy of thyroid stunning. Nuclear Medicine Communications 200425 883-889.

10 Jukkola A, Bloigu R, Ebeling T, Salmela P \& Blanco G. Prognostic factors in differentiated thyroid carcinomas and their implications for current staging classifications. Endocrine-Related Cancer 2004 11 571-579.

11 Giovanella L, Ceriani L, Bhelfo A \& Keller F. Thyroglobulin assay 4 weeks after thyroidectomy predicts outcome in low-risk papillary thyroid carcinoma. Clinical Chemistry and Laboratory Medicine $200543843-847$.

12 Lo CY, Chan WF, Lam KY \& Wan KY. Follicular thyroid carcinoma: the role of histology and staging systems in predicting survival. Annals of Surgery 2005242 707-715.

13 Falvo L, Catania A, Sorrenti S, D'andrea V, Berni A, De Stefano M \& De Antoni E. Prognostic significance of the age factor in the thyroid cancer: statistical analysis. Journal of Surgical Oncology 200488 217-222.

14 Haigh PI, Urbach DR \& Rotstein LE. Extent of thyroidectomy is not a major determinant of survival in low- or high-risk papillary thyroid cancer. Annals of Surgical Oncology 200512 81-89.

15 Cushing SL, Palme CE, Audet N, Eski S, Walfish PG \& Freeman JL. Prognostic factors in well-differentiated thyroid carcinoma. Laryngoscope $20041142110-2115$.

16 Borson-Chazot F, Causeret S, Lifante J, Augros M, Berger N \& Peix J. Predictive factors for recurrence from a series of 74 children and adolescents with differentiated thyroid cancer. World Journal of Surgery 200428 1088-1092.

17 Haq M \& Harmer C. Differentiated thyroid carcinoma with distant metastases at presentation: prognostic factors and outcome. Clinical Endocrinology 200563 87-93.
18 Rodriquez-Cuevas S, Labastida-Almendaro S, Cortes-Arroyo H, Lopez-Garza J \& Barroso-Bravo S. Multifactorial analysis of survival and recurrences in differentiated thyroid cancer. Journal of Experimental and Clinical Cancer Research 200221 79-86.

19 Volante M, Cavallo GP \& Papotti M. Prognostic factors of clinical interest in poorly differentiated carcinomas of the thyroid. Endocrine Pathology 200415 313-317.

20 Siironen P, Louhimo J, Nordling S, Ristimaki A, Maenpaa H, Haapiainen R \& Haglund C. Prognostic factors in papillary thyroid cancer: an evaluation of 601 consecutive patients. Tumor Biology $20052657-64$.

21 Bachelot A, Cailleux AF, Klain M, Baudin E, Ricard M, Bellon N, Caillou B, Travagli JP \& Schlumberger M. Relationship between tumor burden and serum thyroglobulin level in patients with papillary and follicular thyroid carcinoma. Thyroid $2002 \mathbf{1 2}$ 707-711.

22 Bernier MO, Morel O, Rodien P, Muratet JP, Giraud P, Rohmer V, Jeanguillaume C, Bigorgne JC \& Jallet P. Prognostic value of an increase in the serum thyroglobulin level at the time of the first ablative radioiodine treatment in patients with differentiated thyroid carcinoma. European Journal of Nuclear Medicine and Molecular Imaging 200532 1418-1421.

23 Leboulleux S, Rubino C, Baudin E, Caillou B, Hartl DM, Bidart JM, Travagli JP \& Schlumberger M. Prognostic factors for persistent or recurrent disease of papillary thyroid carcinoma with neck lymph node metastases and/or tumor extension beyond the thyroid capsule at initial diagnosis. Journal of Clinical Endocrinology and Metabolism $2005905723-5729$.

24 Hall FT, Beasly NJ, Eski SJ, Witterick IJ, Walfish PG \& Freeman JL. Predictive value of serum thyroglobulin after surgery for thyroid carcinoma. Laryngoscope 2003113 77-81.

25 Baudin E, Do Cao C, Cailleux AF, Leboulleux S, Travagli JP \& Schlumberger M. Positive predictive value of serum thyroglobulin levels, measured during the first year of follow-up after thyroid hormone withdrawal, in thyroid cancer patients. Journal of Clinical Endocrinology and Metabolism 200388 1107-1111.

26 Toubeau M, Touzery C, Arveux P, Chaplain G, Vaillant G, Berriolo A, Riedinger JM, Boichot C, Cochet A \& Brunotte F. Predictive value for disease progression of serum thyroglobulin levels measured in the postoperative period and after (I-131) ablation therapy in patients with differentiated thyroid cancer. Journal of Nuclear Medicine 200445 988-994.

27 Kim TY, Kim WB, Kim ES, Ryu JS, Yeo JS, Kim SC, Hong SJ \& Shong YK. Serum thyroglobulin levels at the time of I-131 remnant ablation just after thyroidectomy are useful of early prediction of clinical recurrence in low-risk patients with differentiated thyroid carcinoma. Journal of Clinical Endocrinology and Metabolism 200590 1440-1445.

Received 20 June 2006

Accepted 31 October 2006 